\title{
Trasporte aéreo de paciente crítico en ECMO
}

Rodrigo Díaz

Médico Jefe Unidad ECMO, Clínica Las Condes, Santiago

El caso clínico presentado por Labbé y cols., en este número de la Revista muestra que, estando en la situación de un shock postcardiotomía, con imposibilidad de salir de bomba, se puede iniciar el soporte in situ con traslado posterior del paciente a un centro de trasplante o uno que tenga más experiencia en soporte vital extracorpóreo. Esto se denomina transporte secundario en ECMO y debemos pensar que es una posibilidad atractiva para nuestro país, donde los "centros ECMO" están concentrados en la Región Metropolitana, V, VIII y IX Regiones, no todos ellos con capacidad de trasplante.
Existen en Chile al menos 19 centros con "máquinas de ECMO", con números desde 0 hasta 55 pacientes por año, con $32 \%$ de los casos acumulados de indicación cardíaca. En el país se hicieron aproximadamente 180 casos de ECMO en el período octubre 2015 a octubre 2016, al menos 20 de ellos fueron de transporte. (Encuesta Nacional de ECMO: datos presentados en Congreso SOCHIMI, Coquimbo Noviembre 2016).

De los 90 pacientes asignados a la rama "ECMO" del estudio CESAR, 2 murieron durante el transporte medicalizado ${ }^{1}$. Poco después de la publicación, el Hospital

Correspondencia: 
de Leicester comenzó con su programa de ECMO móvil. Distintas publicaciones muestran consistentemente que el ECMO de transporte tiene sobrevidas similares a las producidas "in situ", que expande la cobertura de la necesidad de soporte extracorpóreo, que tiene morbilidad asociada limitada y poco frecuente. Eso sí, este tipo de transporte requiere un importante compromiso de equipo, institucional y de políticas públicas ${ }^{2}$.

El soporte vital extracorpóreo ha tenido un aumento exponencial después del año 2009 en la población adulta (coincidiendo con el estudio CESAR y la pandemia AH1N1) ${ }^{3}$. Es muy interesante que este aumento no sólo se vea reflejado en la indicación respiratoria sino a la par, en la indicación cardíaca $^{4}$ (Figura 1).

Figura 1: muestra el número de casos de pacientes adultos reportados a ELSO (Extracorporeal Life Support Organization) hasta julio 2016, los datos mostrados comprenden hasta diciembre 2015.

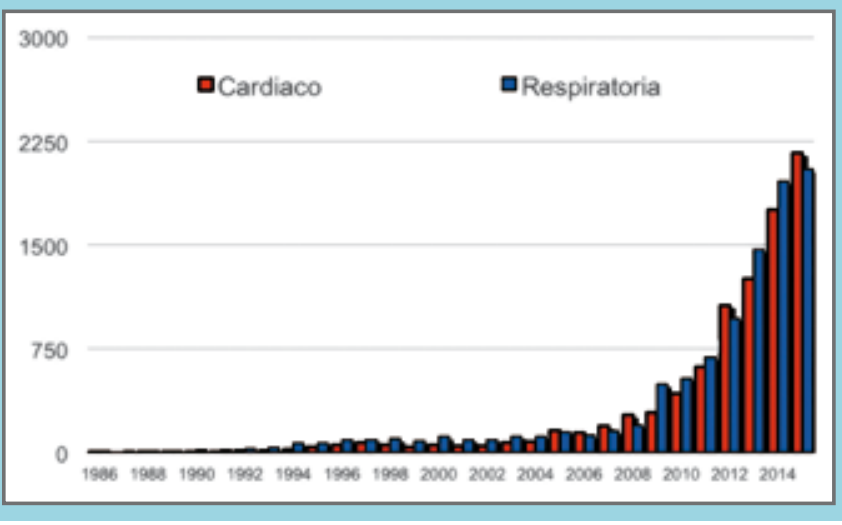

Datos recientes en Alemania, muestran que la tendencia es un aumento de los casos cardíacos sobre los respiratorios. Esto se explica ya que las fallas respiratorias catastróficas con indicación de soporte extracorpóreo son limitadas y la cantidad de pacientes en shock cardiogénico candidatos a soporte mecánico es amplia: un 7\% de los pacientes en shock post infarto con un $40 \%$ de mortalidad y un $1 \%$ de los pacientes en cirugía cardíaca con un $60 \%$ de mortalidad, son al menos potenciales candidatos $5,6,7$.

Los criterios para soporte en falla cardíaca están sugeridos en las Guías clínicas de Insuficiencia Cardíaca de la Sociedad Chilena de Cardiología y Cirugía Cardiovascular y el Ministerio de Salud: "en pacientes seleccionados con IC aguda o crónica severamente descompensados, se recomienda el uso de sistemas de ACM (asistencia circulatoria mecánica) de corta duración". En su algoritmo propuesto son pacientes con 2 o más drogas vasoactivas en dosis altas, que persisten con signos de bajo débito ${ }^{8}$.

En las recomendaciones de la comisión SOCHIMI - MINSAL: "Protocolo operativo para ECMO veno-venoso en falla respiratoria grave", los criterios para considerar rescate por equipo ECMO (no implica necesariamente rescate en ECMO, pero sí rescate por equipo capacitado para transporte en ECMO) son: "Paciente en quien se ha decidido que cumple criterios para derivación a centro con capacidad de ECMO y en que, a pesar de optimización de parámetros ventilatorios, no se consigue $\mathrm{PaO} 2 / \mathrm{FiO} 2>60$. Para pacientes que requieran un traslado desde distancia que requiera avión para su transporte se sugiere emplear límite de $\mathrm{PaO} 2 /$ FiO2 de $80^{9}$.

¿Qué hacer en caso de una falla cardíaca o respiratoria catastrófica, y la gravedad del paciente no permite su traslado y no existe disponible un sistema de soporte extracorpóreo? La alternativa propuesta en este caso clínico parece de toda lógica, si el centro no tiene el entrenamiento, ni la experiencia en el uso de dispositivos de soporte extracorpóreo prolongado, no es razón suficiente para no realizarlo. Es mejor no perder tiempo, instalar el soporte inicial y después transferir al paciente al centro de trasplante que tendrá además mayor experiencia, como en este caso, con el manejo del ECMO. La concentración de casos tiene efectos positivos en lo económico y en los resultados ${ }^{10,11}$.

Debo felicitar a los autores por tan interesante reporte. Este artículo nos muestra que es posible soportar precozmente y trasladar pacientes críticos a centros con mayor experiencia una vez que se logra su estabilización. 


\section{Referencias}

1. PEEK GJ, MUGFORD M, TIRUVOIPATI R, WILSON A, ALLEN E, THALANANY MM, et al. Efficacy and economic assessment of conventional ventilatory support versus extracorporeal membrane oxygenation for severe adult respiratory failure (CESAR): a milticentre randomised controlled trial. Lancet 2009; 374: 1351-63.

2. BRYNER B, COOLEY E, COPENHAVER W, BRIERLEY K, TEMAN N, LANDIS D, et al. Two decades' experience with interfacility transport on extracorporeal membrane oxygenation. The Annals of thoracic surgery, 2014: 98; 1363-1370.

3. ABRAMS D, COMBES A, BRODIE D. Extracorporeal membrane oxygenation in cardiopulmonary disease in adults. Journal of the American College of Cardiology, 2014; 63: 27692778.

4. ELSO (2016) https://www.elso.org/Registry/Statistics/InternationalSummary.aspx. Accedido 10 de Diciembre 2016.

5. KARAGIANNIDIS C, BRODIE D, STRASSMANN S, STOELBEN E, PHILIPP A, BEIN T, et al. Extracorporeal membrane oxygenation: evolving epidemiology and mortality. Intensive care medicine, 2016; 42: 889-896.

6. BABAEV A, FREDERICK PD, PASTA DJ, EVERY N, SICHROVSKY T, HOCHMAN JS, et al. Trends in management and outcomes of patients with acute myocardial infarction complicated by cardiogenic shock. Jama, 2005; 294: 448-454.

7. FUKUHARA S, TAKEDA K, GARAN AR, KURLANSKY P, HASTIE J, NAKA Y, et al. Contemporary mechanical circulatory support therapy for postcardiotomy shock. General thoracic and cardiovascular surgery, 2016; 64: 183-191.

8. http://web.minsal.cl/wp-content/uploads/2015/11/ GUIA-CLINICA-INSUFICIENCIA-CARDIACA_web.pdf Accediido 15 de Diciembre de 2016.

9. GRUPO DE TRABAJO ECMO DE LA SOCIEDAD CHILENA DE MEDICINA INTENSIVA. Protocolo operativo para ECMO veno-venoso en falla respiratoria grave. Revista Chilena de Medicina Intensiva 2015; 30: 75-78.

10. BARBARO RP, ODETOLA FO, KIDWELL KM, PADEN ML, BARTLETT RH, DAVIS MM, \& ANNICH GM. Association of hospital-level volume of extracorporeal membrane oxygenation cases and mortality. Analysis of the extracorporeal life support organization registry. American journal of respiratory and critical care medicine, 2015; 191: 894-901.

11. BECCA J. Regionalization and Triage. ECMO: Extracorporeal Cardiopulmonary Support in Critical Care 4th Edition. 2011. 